\title{
FAIR TOTAL DOMINATION NUMBER IN CACTUS GRAPHS
}

\author{
MAJID HAJIAN \\ Department of Mathematics \\ Shahrood University of Technology \\ Shahrood, Iran \\ e-mail: majid_hajian2000@yahoo.com \\ AND \\ NADER JAFARI RAD \\ Department of Mathematics \\ Shahed University \\ Tehran, Iran \\ e-mail: n.jafarirad@gmail.com
}

\begin{abstract}
For $k \geq 1$, a $k$-fair total dominating set (or just kFTD-set) in a graph $G$ is a total dominating set $S$ such that $|N(v) \cap S|=k$ for every vertex $v \in V \backslash S$. The $k$-fair total domination number of $G$, denoted by $f_{t d}(G)$, is the minimum cardinality of a kFTD-set. A fair total dominating set, abbreviated FTD-set, is a kFTD-set for some integer $k \geq 1$. The fair total domination number of a nonempty graph $G$, denoted by $\operatorname{ftd}(G)$, of $G$ is the minimum cardinality of an FTD-set in $G$. In this paper, we present upper bounds for the 1-fair total domination number of cactus graphs, and characterize cactus graphs achieving equality for the upper bounds.
\end{abstract}

Keywords: fair total domination, cactus graph.

2010 Mathematics Subject Classification: 05C69.

\section{REFERENCES}

[1] Y. Caro, A. Hansberg and M.A. Henning, Fair domination in graphs, Discrete Math. 312 (2012) 2905-2914. doi:10.1016/j.disc.2012.05.006 
[2] B. Chaluvaraju, M. Chellali and K.A. Vidya, Perfect $k$-domination in graphs, Australas. J. Combin. 48 (2010) 175-184.

[3] B. Chaluvaraju and K. Vidya, Perfect dominating set graph of a graph, Adv. Appl. Discrete Math. 2 (2008) 49-57.

[4] E.J. Cockayne, B.L. Hartnell, S.T. Hedetniemi and R. Laskar, Perfect domination in graphs, J. Combin. Inform. System Sci. 18 (1993) 136-148.

[5] I.J. Dejter, Perfect domination in regular grid graphs, Australas. J. Combin. 42 (2008) 99-114.

[6] I.J. Dejter and A.A. Delgado, Perfect domination in rectangular grid graphs, J. Combin. Math. Combin. Comput. 70 (2009) 177-196.

[7] M.R. Fellows and M.N. Hoover, Perfect domination, Australas. J. Combin. 3 (1991) $141-150$.

[8] M. Hajian and N. Jafari Rad, Trees and unicyclic graph with large fair domination number, Util. Math., to appear.

[9] M. Hajian and N. Jafari Rad, Fair domination number in cactus graphs, Discuss. Math. Graph Theory 39 (2019) 489-503. doi: $10.7151 /$ dmgt.2088

[10] M. Hajian, N. Jafari Rad and L. Volkmann, Bounds on the fair total domination number in trees and unicyclic graphs, Australas. J. Combin. 74 (2019) 460-475.

[11] H. Hatami and P. Hatami, Perfect dominating sets in the Cartesian products of prime cycles, Electron. J. Combin. 14 (2007) \#N8. doi:10.37236/1009

[12] T.W. Haynes, S.T. Hedetniemi and P.J. Slater, Fundamentals of Domination in Graphs (Marcel Dekker, New York, 1998).

[13] E.C. Maravilla, R.T. Isla and S.R. Canoy Jr., Fair total domination in the join, corona, and composition of graphs, Int. J. Math. Anal. 8 (2014) 2677-2685.

doi:10.12988/ijma.2014.49296

Received 29 August 2018

Revised 3 April 2019

Accepted 3 April 2019 